\title{
Distributed Output Feedback Control of Decomposable LPV Systems with Delay: Application to Multi-agent Nonholonomic Systems *
}

\author{
Muhammad Zakwan
}

Saeed Ahmed

\begin{abstract}
This paper presents distributed output feedback control of a class of distributed linear parameter varying systems with a parameter-dependent time-varying delay. A delaydependent bounded-real lemma approach, based on parameterdependent Lyapunov-Krasovskii functionals, is used to formulate the synthesis condition in terms of linear matrix inequalities. We demonstrate the efficacy of our result by applying it to formation control of multi-agent nonholonomic mobile robots.

Index Terms-Delay, distributed control, linear parameter varying, linear matrix inequalities
\end{abstract}

\section{INTRODUCTION}

Distributed system is defined as a swarm of subsystems that are interconnected by fictitious or physical interconnections with each subsystem having information of the interconnection topology. There is no necessary condition on dynamical similarity of these subsystems. Automatic control of distributed systems is gaining attention in recent years due to their vast applications that include unmanned aerial vehicles ([2], [24]), large segmented telescopes [14], car platooning [15], and spacecrafts [20].

Decomposable systems is a subclass of distributed systems with identical subsystems interacting in a well-defined pattern modeled by a matrix called the pattern matrix. The framework of decomposable systems for control theoretic applications is proposed in [19]. The definition of the pattern matrix comes from the well known graph theory. In the graph theory paradigm, the subsystems can be represented as nodes of the graph and interconnections can be represented as edges. The Laplacian matrices or graph-adjacency matrices can be used as generalizations of the graph pattern matrix; see [3] and [8] for more details on pattern matrix representations for control theoretic applications. Decomposable systems are ubiquotous in models resulting from the discretization of partial differential equations [5], satellite formation problem [6], paper machine problem [27], and vehicle platooning problem [29].

Time delay appears naturally in applications where there is a non-ideal exchange of the information. A number of contributions in the literature are dedicated to study the effect of time delay in communication networks and how to mitigate it; see, e.g., [1], [12], [13], [16], [17], [21], [22], [23], [25], [26], [28], and [30]. On the other hand, LPV systems can be used to model many real-world applications.

* This work is supported in full by the Science and Research Council of Turkey (TÜBITAK) under project EEEAG-117E948. The authors are with the Department of Electrical and Electronics Engineering, Bilkent University, 06800 Bilkent, Ankara, Turkey. Contact emails: saeed.ahmed@bilkent.edu.tr, seyit.ahmed565@gmail.com
Distributed control of decomposable LPV systems has been studied in [9] using full block S-procedure for distributed controller synthesis. In [9], distributed output feedback controller is synthesized for heterogeneously scheduled decomposable LPV systems in linear fractional (LFT) representation. An application of LPV methods for spatially interconnected systems has been discussed in [7] and [18]; see also [11] and [19] for distributed control of decomposable LTI systems.

In this paper, we study distributed output feedback control of a broad class of decomposable LPV systems with parameter-dependent time-varying communication delay complementing the work of [10], where distributed output feedback control of decomposable LTI systems is discussed with a constant and identical time delay throughout the communication network. In comparison with the global controller design presented in [32], our controller synthesis approach preserves the sparsity structure of the distributed system necessary for a distributed architecture while reducing the computational complexity at the same time. However, our distributed controller architecture comes at the cost of suboptimality as compared to global controller design. To the authors' knowledge, distributed output feedback control of decomposable LPV systems with a parameter-dependent time-varying delay is studied for the first time in this paper. We use a delay-dependent bounded-real lemma approach based on Lyapunov-Krasovskii functionals for formulating synthesis conditions in terms of linear matrix inequalities, ensuring stability and a prescribed level of performance for the decomposable LPV system with parameter-dependent time-varying delay. We also discuss a practical problem of formation control of multi-agent nonholonomic mobile robots to illustrate the effectiveness of our result.

We use standard notation, in which the dimensions of our Euclidean spaces are arbitrary unless otherwise noted, and which will be simplified whenever no confusion would arise. We use $|\cdot|$ to denote the usual Euclidean norm and the induced matrix norm, $I_{n}$ is the identity matrix of dimension $n, O_{n}$ is the null matrix of dimension $n$, and $\otimes$ is the Kronecker product. Let $\mathbb{R}$ represent the set of real numbers, let $\mathbb{R}^{n \times m}$ denote real matrices of dimension $n \times m$, and let $M^{-T}$ denote the inverse of the matrix $M^{T}$. Given any constant $\tau>0$, we let $\mathscr{C}\left([-\tau, 0], \mathbb{R}^{n}\right)$ denote the set of all continuous $\mathbb{R}^{n}$-valued functions that are defined on $[-\tau, 0]$. We call it the set of all initial functions. Also, for any continuous function $x:[-\tau, \infty) \rightarrow \mathbb{R}^{n}$ and all $t \geq 0$, we define $x_{t}$ by $x_{t}(\theta)=x(t+\theta)$ for all $\theta \in[-\tau, 0]$, i.e., $x_{t} \in \phi$ is the translation operator. Let $\mathbb{S}^{n}$ be the set of real 
symmetric matrices of dimension $n \times n, \mathbb{S}_{++}^{n}$ be the set of real symmetric positive definite matrices of dimension $n \times n$, and $\mathbb{S}_{+}^{n}$ be the set of real symmetric semi-positive definite matrices of dimension $n \times n$.

\section{Problem Formulation}

Consider an $N n-t h$ order interconnected LPV system with parameter-dependent delay consisting of $N$ subsystems

$$
\begin{aligned}
\dot{x}(t)= & A_{1}(\rho) x(t)+A_{2}(\rho) x(t-\tau(\rho)) \\
& +B_{1}(\rho) w(t)+B_{2}(\rho) u(t) \\
z(t)= & C_{11}(\rho) x(t)+C_{12}(\rho) x(t-\tau(\rho)) \\
& +D_{11}(\rho) w(t)+D_{12}(\rho) u(t) \\
y(t)= & C_{21}(\rho) x(t)+C_{22}(\rho) x(t-\tau(\rho)) \\
& +D_{21}(\rho) w(t)
\end{aligned}
$$

where $x \in \mathbb{R}^{N n}, w \in \mathbb{R}^{N n_{w}}, u \in \mathbb{R}^{N n_{u}}, z \in \mathbb{R}^{N n_{z}}$, and $y \in \mathbb{R}^{N n_{y}}$ are the state, exogenous input, control input, regulated output, and measured output, respectively. We assume that the exogenous input $w$ is of finite energy in the space $\mathcal{L}_{2}[0 \infty)$, and we assume an initial condition in $\mathscr{C}\left([-\tau, 0], \mathbb{R}^{N n}\right)$. We assume that the time-varying parameter $\rho$ belongs to a set $\mathcal{F}_{\mathscr{P}}^{v}$ defined as $\mathcal{F}_{\mathscr{P}}^{v}:=\left\{\rho \in \mathscr{C}\left(\mathbb{R}_{+}, \mathbb{R}^{s}\right)\right.$ : $\left.\rho(t) \in \mathscr{P},\left|\dot{\rho}_{i}(t)\right| \leq v_{i}\right\}$ where $s$ is the size of parameter varying vector and $\mathscr{P}$ is a compact subset of $\mathbb{R}^{s}$. We assume that the parameter varying delay $\tau$ is differentiable and it belongs to a set $\mathscr{T}$ defined as $\mathscr{T}=\left\{\tau \in \mathscr{C}\left(\mathbb{R}^{s}, \mathbb{R}\right): 0 \leq\right.$ $\left.\tau(t)<\bar{\tau}<\infty, \forall t \in \mathbb{R}_{+}\right\}$. As in [32], we define the worst case performance cost for system (1) from $w$ to $z$ with $u \equiv 0$ as

$$
J=\sup _{\rho \in \mathcal{F}_{\mathscr{P}}^{v}} \sup _{|w| \neq 0} \frac{|z|}{|w|} .
$$

Definition 1: A matrix $M(\rho) \in \mathbb{R}^{N p \times N q}$ is called decomposable if given a diagnonalizable matrix $\mathcal{P}=U \Lambda U^{-1} \in$ $\mathbb{R}^{N \times N}$, there exist matrices $\bar{M}^{a}(\rho), \bar{M}^{b}(\rho) \in \mathbb{R}^{p \times q}$ such that

$$
M(\rho)=I_{N} \otimes \bar{M}^{a}(\rho)+\mathcal{P} \otimes \bar{M}^{b}(\rho)
$$

for all $\rho \in \mathcal{F}_{\mathscr{P}}^{v}$. The superscript $a$ represents the decentralized part and superscript $b$ represents the interconnected part. Moreover, if such a matrix $\mathcal{P}$ is also symmetric, we call the matrix $M(\rho)$ symmetric decomposable.

Assumption 1: We assume that all the system matrices of (1) can be written in the form of (3) for all $\rho \in \mathcal{F}_{\mathscr{P}}^{v}$ and for the same $\mathcal{P}$ where $\mathcal{P}$ is an orthogonal and diagnolizable matrix $\left(U U^{T}=I_{N}\right)$ with real $U$ and $\Lambda$.

We provide some preliminary results that will be used to prove our main result in the next section; see [11] and [19] for LTI corollaries of these results.

Lemma 1: Consider a decomposable matrix $M(\rho)$ of the form (3) as given in Definition 1, then the matrix $M^{\dagger}(\rho)=$ $\left(U \otimes I_{p}\right)^{-1} M(\rho)\left(U \otimes I_{q}\right)$ is block diagonal and has the following structure

$$
M^{\dagger}(\rho)=I_{N} \otimes \bar{M}^{a}(\rho)+\Lambda \otimes \bar{M}^{b}(\rho)
$$

where each of the block has the form $M_{i}^{\dagger}(\rho)=\bar{M}^{a}(\rho)+$ $\lambda_{i} \bar{M}^{b}(\rho)$ where $\lambda_{i}$ is the $i$-th eigenvalue of the matrix $\mathcal{P}$. Moreover, for every matrix $M^{\dagger}(\rho)$ with the structure given in (4), we have $M(\rho)=\left(U \otimes I_{p}\right) M^{\dagger}(\rho)\left(U \otimes I_{q}\right)^{-1}=I_{N} \otimes$ $\bar{M}^{a}(\rho)+\mathcal{P} \otimes \bar{M}^{b}(\rho)$.

Proof: The proof follows straightforwardly using Kronecker product properties.

Theorem 1: If the $N n$-th order LPV system given in (1) satisfies Assumption 1 then it is equivalent to $N$ independent modal subsystems each of order $n$ given by

$$
\begin{aligned}
\dot{\hat{x}}_{i}(t)= & A_{1, i}^{\dagger}(\rho) \hat{x}_{i}(t)+A_{2, i}^{\dagger}(\rho)_{i} \hat{x}_{i}(t-\tau(\rho)) \\
& +B_{1, i}^{\dagger}(\rho) \hat{w}_{i}(t)+B_{2, i}^{\dagger}(\rho) \hat{u}_{i}(t) \\
\hat{z}_{i}(t)= & C_{11, i}^{\dagger}(\rho) \hat{x}_{i}(t)+C_{12, i}^{\dagger}(\rho) \hat{x}_{i}(t-\tau(\rho)) \\
& +D_{11, i}^{\dagger}(\rho) \hat{w}_{i}(t)+D_{12, i}^{\dagger}(\rho) \hat{u}_{i}(t) \\
\hat{y}_{i}(t)= & C_{21, i}^{\dagger}(\rho) \hat{x}_{i}(t)+C_{22, i}^{\dagger}(\rho) \hat{x}_{i}(t-\tau(\rho)) \\
& +D_{21, i}^{\dagger}(\rho) \hat{w}_{i}(t) \text { for } i=1,2, \ldots, N
\end{aligned}
$$

where $\hat{x}_{i} \in \mathbb{R}^{n}, \hat{w}_{i} \in \mathbb{R}^{n_{w}}, \hat{u}_{i} \in \mathbb{R}^{n_{u}}, \hat{z}_{i} \in \mathbb{R}^{n_{z}}$, and $\hat{y}_{i} \in$ $\mathbb{R}^{n_{y}}$ are the state, exogenous input, control input, regulated output, and measured output of the $i$-th modal subsystem, respectively. Moreover, the matrices $A_{1, i}^{\dagger}, A_{2, i}^{\dagger}, \ldots, D_{21, i}^{\dagger}$ are defined as

$$
\begin{aligned}
A_{1, i}^{\dagger}(\rho) & =\bar{A}_{1}^{a}(\rho)+\lambda_{i} \bar{A}_{1}^{b}(\rho) \\
A_{2, i}^{\dagger}(\rho) & =\bar{A}_{2}^{a}(\rho)+\lambda_{i} \bar{A}_{2}^{b}(\rho) \\
& \vdots \\
D_{21, i}^{\dagger}(\rho) & =\bar{D}_{21}^{a}(\rho)+\lambda_{i} \bar{D}_{21}^{b}(\rho)
\end{aligned}
$$

where $\lambda_{i}$ is the $i$-th eigenvalue of the matrix $\mathcal{P}$.

Proof: The proof follows using similar arguments found in [11], [19], and [32] along with the following similarity transformations

$$
\begin{aligned}
x(t) & =\left(U \otimes I_{n}\right) \hat{x}(t) \\
w(t) & =\left(U \otimes I_{n_{w}}\right) \hat{w}(t) \\
u(t) & =\left(U \otimes I_{n_{u}}\right) \hat{u}(t) \\
z(t) & =\left(U \otimes I_{n_{z}}\right) \hat{z}(t) \\
y(t) & =\left(U \otimes I_{n_{y}}\right) \hat{y}(t),
\end{aligned}
$$

and Lemma 1.

In the next section, we drop the dependence on $\rho$ in the notation for the sake of simplicity of the presentation.

\section{Delay-Dependent Distributed Controller SYNTHESIS}

In this section, we design distributed output feedback controller for decomposable LPV systems with a parameterdependent time-varying delay using the idea of [32]. Our aim is to synthesize output feedback controller in the following distributed architecture form

$$
\begin{aligned}
\dot{x}_{k}(t) & =\mathcal{M}_{1} x_{k}(t)+\mathcal{M}_{2} x_{k}(t-\tau)+\mathcal{B} y(t) \\
u(t) & =\mathcal{C}_{11} x_{k}(t)+\mathcal{C}_{12} x_{k}(t-\tau)+\mathcal{D} y(t)
\end{aligned}
$$

where $x_{k}$ is valued in $\mathbb{R}^{N n}$, and

$$
\begin{aligned}
\mathcal{M}_{1} & =I_{N} \otimes \overline{\mathcal{M}}^{a} \\
\mathcal{M}_{2} & =\mathcal{P} \otimes \overline{\mathcal{M}}^{b} \\
\mathcal{B} & =I_{N} \otimes \overline{\mathcal{B}}^{a} \\
\mathcal{C}_{11} & =I_{N} \otimes \overline{\mathcal{C}}^{a} \\
\mathcal{C}_{12} & =\mathcal{P} \otimes \overline{\mathcal{C}}^{b} \\
\mathcal{D} & =I_{N} \otimes \overline{\mathcal{D}}^{a} .
\end{aligned}
$$


Since the controller has a distributed architecture, we can use similar arguments as in the proof of Theorem 1 to decompose the controller given in (7) into $N$ controllers: one corresponding to each modal subsystem in (5). The structure of the controller corresponding to the $i^{t h}$ modal subsystem in (5) is given as

$$
\begin{aligned}
\dot{\hat{x}}_{k, i}(t)= & \mathcal{M}_{1, i}^{\dagger} \hat{x}_{k, i}(t)+\mathcal{M}_{2, i}^{\dagger} \hat{x}_{k, i}(t-\tau) \\
& +\mathcal{B}_{i}^{\dagger} \hat{y}(t) \\
\hat{u}_{i}(t)= & \mathcal{C}_{11, i}^{\dagger} \hat{x}_{k, i}(t)+\mathcal{C}_{12, i}^{\dagger} \hat{x}_{k, i}(t-\tau) \\
& +\mathcal{D}_{i}^{\dagger} \hat{y}(t)
\end{aligned}
$$

for $i=1,2, \ldots, N$.

Assumption 2: We assume that $\bar{D}_{12}^{b}=0, \bar{C}_{21}^{b}=0, \bar{C}_{22}^{b}=$ $0, \bar{B}_{2}^{b}=0$, and $\bar{D}_{21}^{b}=0$.

This assumption is made to avoid multiplication of $\lambda_{i}$ 's in constructing the controller parameters. This is a widely used assumption in the literature for full-order output feedback control of decomposable systems; see [10], [11], and [19].

Theorem 2: Assume that the interconnected LPV system with a parameter-dependent time-varying delay described by (1) satisfies Assumptions 1 and 2. A sufficient condition for the existence of a distributed dynamic output feedback controller that guarantees asymptotic stability of the closedloop system and minimizes the performance cost i.e., $J<\gamma$ is that the set of LMIs $(\star)$ are satisfied for $i=1,2, \ldots, N$ and some continuously differentiable matrix functions $X, Y$ : $\mathbb{R}^{s} \rightarrow \mathbb{S}_{++}^{n}$, where

$$
\begin{aligned}
& \tilde{\mathscr{V}}=\left[\begin{array}{cc}
Y & I_{n} \\
I_{n} & X
\end{array}\right] \\
& \mathscr{A}_{1, i}=\left[\begin{array}{cc}
A_{1, i}^{\dagger} Y+\bar{B}_{2}^{a} \hat{C}_{a} & A_{1, i}^{\dagger}+\bar{B}_{2}^{a} \mathcal{R} C_{21, i}^{\dagger} \\
\hat{A}^{a} & X A_{1, i}^{\dagger}+\hat{B} C_{21, i}^{\dagger}
\end{array}\right] \\
& \mathscr{A}_{2, i}=\left[\begin{array}{cc}
A_{2, i}^{\dagger} Y+\bar{B}_{2}^{a} \hat{C}_{b} & A_{2, i}^{\dagger}+\bar{B}_{2}^{a} \mathcal{R} C_{22, i}^{\dagger} \\
\lambda_{i} \hat{A}^{b} & X A_{2, i}^{\dagger}+\hat{B} C_{22, i}^{\dagger}
\end{array}\right] \\
& \mathscr{B}_{i}=\left[\begin{array}{c}
B_{1, i}^{\dagger}+\bar{B}_{2}^{a} \mathcal{R} D_{21, i}^{\dagger} \\
X B_{1, i}^{\dagger}+\hat{B} D_{21, i}^{\dagger}
\end{array}\right] \\
& \mathscr{C}_{11, i}=\left[C_{11, i}^{\dagger} Y+\bar{D}_{12}^{a} \hat{C}^{a} \quad C_{11, i}^{\dagger}+\bar{D}_{12}^{a} \mathcal{R} C_{21, i}^{\dagger}\right] \\
& \mathscr{C}_{12, i}=\left[C_{12, i}^{\dagger} Y+\lambda_{i} \bar{D}_{12}^{a} \hat{C}^{b} \quad C_{12, i}^{\dagger}+\bar{D}_{12}^{a} \mathcal{R} C_{22, i}^{\dagger}\right] \\
& \mathscr{D}_{i}=\left[D_{11, i}^{\dagger}+\bar{D}_{12}^{a} \mathcal{R} D_{21, i}^{\dagger}\right] \\
& \tilde{\Psi}_{22}=\tilde{\mathscr{Q}}-\tilde{\mathscr{R}}+\left[\sum_{j=1}^{s} \pm\left(v_{j} \frac{\partial \tilde{\mathscr{P}}(\rho)}{\partial \rho_{j}}\right)\right] \\
& \tilde{\Theta}_{22}=-\left[1-\sum_{j=1}^{s} \pm\left(v_{j} \frac{\partial \tau}{\partial \rho_{j}}\right)\right] \tilde{\mathscr{Q}}-\tilde{\mathscr{R}},
\end{aligned}
$$

$\tilde{\mathscr{P}}: \mathbb{R}^{s} \rightarrow \mathbb{S}_{++}^{2 n}$ and $\tilde{\mathscr{Q}}, \tilde{\mathscr{R}} \in \mathbb{S}_{++}^{2 n}$ are constant matrices, $\hat{A}^{a}, \hat{A}^{b}, \hat{B}, \hat{C}^{a}, \hat{C}^{b}$ and $\mathcal{R}$ are $\rho$-dependent matrices, and $\gamma>$ 0 is a scalar. Moreover, if LMIs have feasible solutions, the controller matrices appearing in (7) and (8) are given by

$$
\begin{aligned}
\overline{\mathcal{M}}^{a}= & -N^{-1}\left(X \bar{A}_{1}^{a} Y+X \bar{B}_{2}^{a} \mathcal{R} \bar{C}_{21}^{a} Y\right. \\
& \left.+N \overline{\mathcal{B}}^{a} \bar{C}_{21}^{a} Y+X \bar{B}_{2}^{a} \overline{\mathcal{C}}^{a} M^{T}-\hat{A}^{a}\right) M^{-T} \\
\overline{\mathcal{M}}^{b}= & -N^{-1}\left(X \bar{A}_{2}^{b} Y+X \bar{B}_{2}^{a} \mathcal{R} \bar{C}_{21}^{b} Y\right. \\
& \left.+N \overline{\mathcal{B}}^{a} \bar{C}_{21}^{b} Y+X \bar{B}_{2}^{a} \overline{\mathcal{C}}^{b} M^{T}-\hat{A}^{b}\right) M^{-T} \\
\overline{\mathcal{B}}^{a}= & N^{-1}\left(\hat{B}-X \bar{B}_{2}^{a} \mathcal{R}\right) \\
\overline{\mathcal{C}}^{a}= & \left(\hat{C}^{a}-\mathcal{R} \bar{C}_{21}^{a} Y\right) M^{-T} \\
\overline{\mathcal{C}}^{b}= & \left(\hat{C}^{b}-\mathcal{R} \bar{C}_{21}^{b} Y\right) M^{-T} \\
\overline{\mathcal{D}}^{a}= & \mathcal{R}
\end{aligned}
$$

where $M$ and $N$ are obtained by factorization problem $I_{n}-$ $X Y=N M^{T}$.

The proof of Theorem 2 follows directly from our journal version [31].

\section{Formation Control of Nonholonomic Mobile ROBOTS}

To illustrate the efficacy of our result, we provide a practical example of formation control of multi-agent nonholonomic mobile robots. Consider a multi-agent formation setting where we have $N=4$ nonholonomic mobile robots (agents) each of order $n=2$ such that each agent can communicate with its adjacent agent on both of its side as depicted in Figure 1. We assume that these agents are uniformly distributed in a circular manner and our aim is to design a distributed LPV controller that minimizes the error on their relative positions under the performance criterion given by (2). Moreover, we consider a parameter-dependent time-varying communication delay $\tau(\rho)$ among these agents. The distributed controller will stabilize the system with these patterns while minimizing the following relative position error

$$
z_{x_{j}, i}=-\frac{1}{2} x_{j, i-1}+x_{j, i}-\frac{1}{2} x_{j, i+1}
$$

for $j=1, \ldots, n$ and $i=1,2, \ldots, N$. As there is no dynamic interaction among these agents, all matrices in (1) will be block diagonal except $C_{12}$ which has the structure $\mathcal{P} \otimes \bar{C}_{12}^{b}$. We can represent the multi-agent non-holonomic system as the following delayed decomposable LPV system

$$
\begin{aligned}
\dot{x}(t)= & \left(I_{N} \otimes A_{1}(\rho)\right) x(t)+\left(\mathcal{P} \otimes A_{2}(\rho)\right) x(t-\tau(\rho)) \\
& +\left(I_{N} \otimes B_{1}(\rho)\right) w(t)+\left(I_{N} \otimes B_{2}(\rho)\right) u(t) \\
z(t)= & \left(I_{N} \otimes C_{11}(\rho)\right) x(t)+\left(\mathcal{P} \otimes C_{12}(\rho)\right) x(t-\tau(\rho)) \\
& +\left(I_{N} \otimes D_{11}(\rho)\right) w(t)+\left(I_{N} \otimes D_{12}(\rho)\right) u(t) \\
y(t)= & \left(I_{N} \otimes C_{21}(\rho)\right) x(t)+\left(\mathcal{P} \otimes C_{22}(\rho)\right) x(t-\tau(\rho)) \\
& +\left(I_{N} \otimes D_{21}(\rho)\right) w(t)
\end{aligned}
$$

with $x, w, u, z$, and $y$ all valued in $\mathbb{R}^{8}, \rho=\left[\rho_{1}, \rho_{2}\right]$, and the system matrices are given by

$$
\begin{gathered}
A_{1}(\rho)=\left[\begin{array}{cc}
0 & \rho_{1} \\
\rho_{1} & 0
\end{array}\right], A_{2}(\rho)=O_{2}, B_{1}=B_{2}=\left[\begin{array}{cc}
1 & 0 \\
0 & 0.1
\end{array}\right], \\
C_{11}=C_{12}=C_{21}=I_{2}, C_{22}=O_{2} \\
D_{11}=0, D_{12}=0.1 I_{2}, D_{21}=0 .
\end{gathered}
$$




$$
\Psi_{i}(\rho):=\left[\begin{array}{cccccc}
-2 \tilde{\mathscr{V}} & \tilde{\mathscr{P}}-\tilde{\mathscr{V}}+\mathscr{A}_{1, i} & -\tilde{\mathscr{V}}+\mathscr{A}_{2, i} & \mathscr{B}_{i} & 0 & \tilde{\mathscr{V}}+\bar{\tau} \tilde{\mathscr{R}} \\
* & \tilde{\Psi}_{22}+\mathscr{A}_{1, i}+\mathscr{A}_{i}^{T} & \tilde{\mathscr{R}}+\mathscr{A}_{1, i}^{T}+\mathscr{A}_{2, i} & \mathscr{B}_{i} & \mathscr{C}_{11, i}^{T} & \tilde{\mathscr{V}}-\tilde{\mathscr{P}} \\
* & * & \tilde{\Theta}_{22}+\mathscr{A}_{2, i}+\mathscr{A}_{2, i}^{T} & \mathscr{B}_{i} & \mathscr{C}_{12, i}^{T} & \tilde{\mathscr{V}} \\
* & * & * & -\gamma I_{n_{w}} & \mathscr{D}_{i}^{T} & 0 \\
* & * & * & * & -\gamma I_{n_{z}} & 0 \\
* & * & * & * & * & (-1-2 \bar{\tau}) \tilde{\mathscr{R}}
\end{array}\right]<0, \forall \rho \in \mathcal{F}_{\mathscr{P}}^{v}
$$

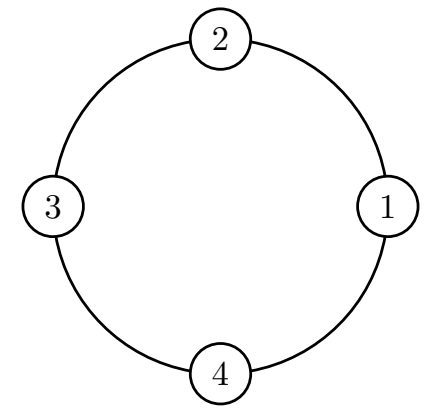

Fig. 1. Formation of nonholonomic multi-agent system

We choose $\rho_{1}=\omega \sin (t)$ and $\rho_{2}=|\cos (\omega t)|$ with $v_{1}=$ $v_{2}=5, \tau(\rho)=\mu \rho_{2}, \mu=0.09$, and $\omega=5 \mathrm{rad} / \mathrm{sec}$.

For the formation problem of four agents depicted in Fig. 1 , the pattern matrix is given below

$$
\mathcal{P}=U \Lambda U^{T}=\left[\begin{array}{cccc}
1.0 & -0.5 & 0.0 & -0.5 \\
-0.5 & 1.0 & -0.5 & 0.0 \\
0.0 & -0.5 & 1.0 & -0.5 \\
-0.5 & 0.0 & -0.5 & 1.0
\end{array}\right]
$$

with

$$
\begin{gathered}
U=\left[\begin{array}{cccc}
0.5 & 0.7071 & 0.0 & -0.5 \\
0.5 & 0 & 0.7071 & 0.5 \\
0.5 & -0.7071 & 0 & -0.5 \\
0.5 & 0 & -0.7071 & 0.5
\end{array}\right] \\
\Lambda=\left[\begin{array}{llll}
0 & 0 & 0 & 0 \\
0 & 1 & 0 & 0 \\
0 & 0 & 1 & 0 \\
0 & 0 & 0 & 2
\end{array}\right]
\end{gathered}
$$

The controller is designed such that the agents reach a consensus by solving the LMIs in $(\star)$. The parameter space

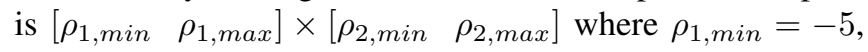
$\rho_{1, \max }=5, \rho_{2, \min }=0$, and $\rho_{2, \max }=1$. The time delay $\tau$ varies from 0 to $\bar{\tau}=0.09$, and the condition $d \tau / d t<1$ holds except for a countable number of points. Moreover, $\left|d \rho_{1} / d t\right| \leq 5$ and $\left|d \rho_{2} / d t\right| \leq 5$, hence the controller does not depend on future value of the parameters. Since the system matrix $A_{1}(\rho)$ has affine dependence on only $\rho_{1}$ and $A_{2}(\rho)=$ $\mathrm{O}_{2}$, we can design LPV controller (7) for the system (12) in polytopic form using two vertices of $\rho_{1}$ i.e., $\left[\begin{array}{lll}\rho_{1, \min } & \rho_{1, \max }\end{array}\right]$. The matrices $\overline{\mathcal{M}}^{a}$ and $\overline{\mathcal{M}}^{b}$ in (8) are given by

$$
\begin{aligned}
& \overline{\mathcal{M}}^{a}\left(\rho_{1}\right)=c_{1}\left(\rho_{1}\right) \overline{\mathcal{M}}_{\text {max }}^{a}+c_{2}\left(\rho_{1}\right) \overline{\mathcal{M}}_{\text {min }}^{a} \\
& \overline{\mathcal{M}}^{b}\left(\rho_{1}\right)=c_{1}\left(\rho_{1}\right) \overline{\mathcal{M}}_{\text {max }}^{b}+c_{2}\left(\rho_{1}\right) \overline{\mathcal{M}}_{\text {min }}^{b}
\end{aligned}
$$

where

$$
\begin{aligned}
\overline{\mathcal{M}}_{\text {max }}^{a} & =\left[\begin{array}{cc}
-3.9354 & 0.7298 \\
-2.1356 & -3.2729
\end{array}\right] \\
\overline{\mathcal{M}}_{\text {min }}^{a} & =\left[\begin{array}{cc}
-4.0194 & -0.8327 \\
2.5501 & -3.2154
\end{array}\right] \\
\overline{\mathcal{M}}_{\text {max }}^{b} & =\left[\begin{array}{cc}
-0.1151 & 0.0198 \\
-0.1119 & 0.1341
\end{array}\right] \\
\overline{\mathcal{M}}_{\text {min }}^{b} & =\left[\begin{array}{cc}
-0.1901 & -0.0042 \\
0.0939 & 0.1552
\end{array}\right] \\
c_{1}\left(\rho_{1}\right) & =\frac{\rho_{1, \max }-\rho_{1}}{\rho_{1, \max }-\rho_{1, \min }} \\
c_{2}\left(\rho_{1}\right) & =\frac{\rho_{1}-\rho_{1, \min }}{\rho_{1, \max }-\rho_{1, \min }} \\
c_{1}\left(\rho_{1}\right)+c_{2}\left(\rho_{1}\right) & =1,
\end{aligned}
$$

and the matrices $\overline{\mathcal{B}}^{a}, \overline{\mathcal{C}}^{a}, \overline{\mathcal{C}}^{b}$, and $\overline{\mathcal{D}}^{a}$ in (8) are

$$
\begin{aligned}
\overline{\mathcal{B}}^{a} & =\left[\begin{array}{cc}
-0.0044 & -0.0000 \\
0.0009 & -0.0075
\end{array}\right] \\
\overline{\mathcal{C}}^{a} & =\left[\begin{array}{cc}
-0.7727 & 0.0246 \\
4.4675 & 4.5136
\end{array}\right] \\
\overline{\mathcal{C}}^{b} & =\left[\begin{array}{cc}
0.6482 & -0.0016 \\
-0.0795 & -1.9060
\end{array}\right] \\
\overline{\mathcal{D}}^{a} & =\left[\begin{array}{ll}
-3.7087 & -0.0047 \\
-0.0047 & -25.1093
\end{array}\right] .
\end{aligned}
$$

Fig. 2 illustrates the stability and consensus of all four agents in closed-loop configuration for different initial conditions under a step disturbance. The simulations corroborate overall system stability under parameter-dependent timevarying delay.

\section{CONCLUSIONS}

We proposed synthesis conditions for distributed dynamic output feedback controller for a broad class of decomposable LPV systems with a parameter-dependent time-varying delay which ensures asymptotic stability as well as satisfactory performance. We also provided the application of our result to formation control of multi-agent nonholonomic mobile robots. Many extensions of our result are possible; for instance, extension to switching and time varying topologies, extension to other families of distributed systems and 


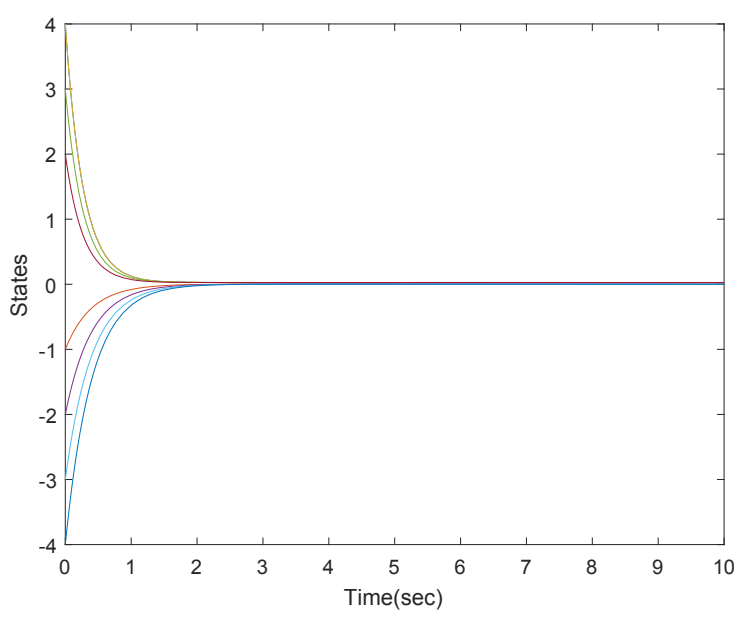

Fig. 2. Closed-loop state trajectories under a step disturbance

coordination patterns, and extension to the case where the parameter variation is stochastic as in [4].

\section{ACKNOWLEDGMENT}

We would like to thank Prof. Hitay Özbay for his insightful comments which helped in improving the quality of our paper.

\section{REFERENCES}

[1] F. M. Atay, "The consensus problem in networks with transmission delays," Philosophical Transactions of the Royal Society A, vol. 371, no. 1999, 2013.

[2] A. Betser, P. A. Vela, G. Pryor, and A. Tannenbaum, "Flying in formation using a pursuit guidance algorithm," in Proceedings of the American Control Conference, 2005, pp. 5085 - 5090.

[3] F. Borrelli and T. Keviczky, "Distributed LQR design for identical dynamically decoupled systems," IEEE Transactions on Automatic Control, vol. 53, no. 8, pp. 1901 - 1912, 2008.

[4] C. Briat, "Stability analysis and state-feedback control of LPV systems with piecewise constant parameters subject to spontaneous poissonian jumps," IEEE Control Systems Letters, vol. 2, no. 2, pp. 230 - 235, 2018.

[5] R. W. Brockett and J. L. Willems, "Discretized partial differential equations: Example of control systems defined on modules," Automatica, vol. 10, no. 5, pp. 507 - 515, 1974.

[6] J. R. Carpenter, "A preliminary invetigation of decentralized control for satellite formations," in Proceedings of the IEEE Aerospace Conference, 2000, pp. $63-74$.

[7] S. S. Chughtai and H. Werner, "Modeling and distributed control of transition in plane poiseuille flow," IEEE Transactions on Control Systems Technology, vol. 20, no. 3, pp. 755 - 762, 2012.

[8] R. Diestel, Graph Theory. Berlin: Springer, 1996.

[9] A. Eichler, C. Hoffmann, and H. Werner, "Robust control of decomposable LPV systems," Automatica, vol. 50, no. 12, pp. 3239 - 3245, 2014.

[10] R. Ghadami, "Distributed control of multi-agent systems with switching topology, delay, and link failure," Doctoral Dissertation, Northeastern University, Boston, MA, 2012.

[11] R. Ghadami and B. Shafai, "Decomposition-based distributed control for continuous-time multi-agent systems," IEEE Transactions on Automatic Control, vol. 58, no. 1, pp. 258 - 264, 2013.

[12] Y. Ghaedsharaf, M. Siami, C. Somarakis, and N. Motee, "Interplay between performance and communication delay in noisy linear consensus networks," in Proceedings of the European Control Conference, 2016, pp. 1703 - 1708 .

[13] J. Hu and Y. S. Lin, "Consensus control for multi-agent systems with double-integrator dynamics and time delays," IET Control Theory and Applications, vol. 4, no. 1, pp. 109 - 118, 2010.
[14] S. Jiang, P. G. Voulgaris, L. E. Holloway, and L. A. Thompson, "Distributed control of large segmented telescopes," in Proceedings of the American Control Conference, 2006, pp. 1942 - 1947.

[15] M. R. Jovanovic and B. Bamieh, "On the ill-posedness of certain vehicular platoon control problems," IEEE Transactions on Automatic Control, vol. 50, no. 9, pp. 1307 - 1321, 2005.

[16] P. Lin and Y. Jia, "Average consensus in networks of multi-agents with both switching topology and coupling time-delay," Physica A: Statistical Mechanics and its Applications, vol. 387, no. 1, pp. $303-$ 313, 2008.

[17] C. Liu and F. Liu, "Consensus problem of second-order multiagent systems with time-varying communication delay and switching topology," Journal of Systems Engineering and Electronics, vol. 22, no. 4, pp. $672-678,2011$.

[18] Q. Liu, C. Hoffman, and H. Werner, "Distributed control of parametervarying spatially interconnected systems using parameter-dependent Lyapunov functions," in Proceedings of the American Control Conference, 2013, pp. $3278-3283$.

[19] P. Massioni and M. Verhaegen, "Distributed control for identical dynamically coupled systems: A decomposition approach," IEEE Transactions on Automatic Control, vol. 54, no. 1, pp. 124 - 135 , 2009.

[20] M. Mesbahi and F. Y. Hadaegh, "Formation flying control of multiple spacecraft via graphs, matrix inequalities, and switching," Journal of Guidance, Control, and Dynamics, vol. 24, no. 2, pp. 369 - 377, 2001.

[21] R. Olfati-Saber and R. M. Murray, "Consensus problems in networks of agents with switching topology and time-delays," IEEE Transactions on Automatic Control, vol. 49, no. 9, pp. 1520 - 1533, 2004.

[22] A. Papachristodoulou, A. Jadbabaie, and U. Munz, "Effects of delay in multi-agent consensus and oscillator synchronization," IEEE Transactions on Automatic control, vol. 55, no. 6, pp. 1471 - 1477, 2010.

[23] L. Peng, J. Yingmin, D. Junping, and Y. Shiying, "Distributed consensus control for second-order agents with fixed topology and timedelay," in Proceedings of the Chinese Control Conference, 2007, pp. $577-581$.

[24] U. Pilz, A. Popov, and H. Werner, "An information flow filter approach to cooperative vehicle control and its application to formation flight of quad-rotor helicopters," Asian Journal of Control, 2012.

[25] W. Qiao and R. Sipahi, "Consensus control under communication delay in a three-robot system: Design and experiments," IEEE Transactions on Control Systems Technology, vol. 24, no. 2, pp. 687 - 694 2016.

[26] A. Seuret, D. V. Dimarogonas, and K. H. Johansson, "Consensus under communication delays," in Proceedings of the 47th IEEE Conference on Decision and Control, 2008, pp. 4922 - 4927.

[27] G. E. Stewart, D. M. Gorinevsky, and G. A. Dumont, "Feedback controller design for a spatially distributed system: the paper machine problem," IEEE Transactions on Control Systems Technology, vol. 11 no. 5, pp. 612 - 628, 2003.

[28] Y. G. Sun and L. Wang, "Consensus of multi-agent systems in directed networks with nonuniform time-varying delays," IEEE Transactions on Automatic Control, vol. 54, no. 7, pp. 1607 - 1613, 2009.

[29] M. J. Woo and J. W. Choi, "A relative navigation system for vehicle platooning," in Proceedings of the 40th SICE Annual Conference, 2001, pp. 28-31

[30] B. Yang, H. Fang, and H. Wang, "Second-order consensus in networks of dynamic agents with communication time-delays," Journal of Systems Engineering and Electronics, vol. 21, no. 1, pp. 88 - 94 , 2010.

[31] M. Zakwan and S. Ahmed, "Distributed output feedback control of decomposable LPV systems with delay and switching topology: Consensus in multi-agent nonholonomic systems," 2019, submitted.

[32] R. Zope, J. Mohammadpour, K. Grigoriadis, and M. Franchek, "Delaydependent output feedback control of time-delay LPV systems," in Control of Linear Parameter Varying Systems with Applications. Boston: Springer, 2012, pp. 279 - 299. 